\title{
Existence Theorems for the Dirichlet Elliptic Inclusion Involving Exponential-Growth-Type Multivalued Right-Hand Side
}

\author{
by \\ Hôǹg Thái NGUYỄ̃ and Dariusz PĄCZKA
}

Presented by Czesław OLECH

\begin{abstract}
Summary. We present two existence results for the Dirichlet elliptic inclusion with an upper semicontinuous multivalued right-hand side in exponential-type Orlicz spaces involving a vector Laplacian, subject to Dirichlet boundary conditions on a domain $\Omega \subset \mathbb{R}^{2}$. The first result is obtained via the multivalued version of the Leray-Schauder principle together with the Nakano-Dieudonné sequential weak compactness criterion. The second result is obtained by using the nonsmooth variational technique together with a formula for Clarke's subgradient for Lipschitz integral functionals on "nonregular" Orlicz spaces.
\end{abstract}

1. Introduction. Let $\Omega \subset \mathbb{R}^{2}$ be an open bounded domain. We establish two results (see Theorems 3.1 and 5.1) for the Dirichlet elliptic inclusion on $\Omega$ involving an upper semicontinuous multivalued right-hand side with exponential-growth-type conditions (in connection with PokhozhaevTrudinger's theorem on the exact embedding of the Sobolev space $H_{0}^{1}(\Omega)$ into the Orlicz space $L_{\Phi_{0}}(\Omega)$ with $\left.\Phi_{0}(t)=\exp \left(t^{2}\right)-1\right)$. The proof of Theorem 3.1 is based on the multivalued version of the Leray-Schauder principle (see, e.g., $[2,3]$ ) and on the Nakano-Dieudonné sequential $\sigma(Y, Z)$-weak compactness and completeness criteria $[14,8,31]$ together with [28, Theorem 2.1]. Note that Theorem 2.1 of [28] can be regarded as a generalization

2000 Mathematics Subject Classification: Primary 35R70, 49J52, 46E30; Secondary 47H04, 47H30, 54C60, 49J53.

Key words and phrases: Dirichlet elliptic inclusion, exponential-growth-type multivalued right-hand side, weak solutions in Orlicz spaces, topological and non-smooth variational techniques, generalized gradient, subgradient in Clarke's sense, Lipschitz integral functional. 
of the strong-weak closedness theorem from the work of C. Olech, A. Lasota, L. Cesari, C. Castaing and others (for references, see e.g., [21]). The proof of Theorem 5.1 relies on a generalization of the non-smooth variational technique [11] (see also [19, 25]) but via using a formula (see Theorem 4.3) for Lipschitz integral functionals defined on a "nonregular" Orlicz space $L_{\Phi}(\Omega)$. Observe that the formula of this type in [32, Theorem 2] cannot be applied to the above problem, since it holds only for integral functionals defined on regular Orlicz spaces $L_{\Psi}$ (i.e., with $\Psi$ satisfying the $\Delta_{2}$-condition).

Many results for the Dirichlet elliptic inclusion were obtained via different techniques (see $[2,3,6,7,9,11,18,19,21,24,25,29,30,33]$ ). In fact, Theorem 3.1 gives a new sufficient solvability condition for the application of [2, Theorem 2]. Existence results in exponential-type Orlicz spaces for the Dirichlet inclusion involving a lower semicontinuous multivalued right-hand side were obtained in [29]. Theorem 5.1 is an $L_{\Phi^{-}}$generalization of the scheme of [11] (see also [25]) where the Dirichlet elliptic inclusion had a multivalued right-hand side with polynomial-growth-type conditions and Clarke's formula in the Lebesgue spaces $L_{p}(\Omega)$ was used $[12,13]$ (cf. [11]).

Other non-smooth variational techniques (see [19, 25] and the references therein) can also be generalized (by using arguments analogous to those in the proof of Theorem 5.1) and applied to the Dirichlet elliptic inclusion with multivalued right-hand side satisfying exponential-growth-type conditions. We shall present these generalizations in our subsequent paper which uses the formula of Theorem 4.3. Observe that Theorem 4.3 can also be generalized to functionals defined on Banach lattices, non-solid generalized Orlicz spaces, and Banach $M$-spaces (for the definitions, see respectively [5], $[22,23,26]$, and [27]).

2. Some terminology and notation. Our terminology and notation from set-valued analysis follows [3, 4, 10, 18], from function space theory follows [5, 26, 34], and from nonsmooth analysis follows [13, 24]. Throughout this paper $E$ denotes a separable Banach space, and $E_{w^{*}}^{*}$ its dual space endowed with the weak topology $w^{*}=\sigma\left(E^{*}, E\right)$. Put $B_{E}(u, r):=\{\omega \in E$ : $\left.\|\omega-u\|_{E} \leq r\right\}$ for $r \in(0, \infty)$ and let Int $B_{E}(u, r)$ denote the corresponding open ball. Given a Suslin locally convex space $F$ (e.g. $F=E$ or $F=E_{w^{*}}^{*}$ ) [10], we denote by $\mathcal{B}(F)$ the $\sigma$-algebra of Borel subsets of $F$, and by $\operatorname{Cp}(F)$ (resp., $\operatorname{CvCp}(F)$ ) the family of all nonempty compact (resp., convex compact) subsets of $F$. A multifunction $\Gamma: \Omega \rightarrow 2^{F}$ is called Sel-measurable if Sel $\Gamma \neq \emptyset$ where Sel $\Gamma$ denotes the set of all measurable selections of $\Gamma$. Given a function $\alpha: \Omega \rightarrow E$ and a multifunction $H: \Omega \times E \rightarrow \operatorname{Cp}\left(E_{\omega^{*}}^{*}\right)$, define the multivalued superposition

$$
N_{H}(\alpha):=\operatorname{Sel} H(\cdot, \alpha(\cdot)) .
$$


Let $X, Y$ be metric spaces and let $\Gamma: X \rightarrow 2^{Y} \backslash\{\emptyset\}$ be a multifunction. Then $\Gamma$ is called closed if its graph $\operatorname{Gr} \Gamma=\{[x, y] \in X \times Y: y \in \Gamma(x)\}$ is a closed subset of $X \times Y$; locally compact (resp., locally bounded) if each point $x \in X$ has a neighborhood $U$ such that $\overline{\Gamma(U)}$ is compact (resp., bounded); upper semicontinuous (or u.s.c.) at $x \in X$ if, for any open set $V \subset Y$ with $\Gamma(x) \subseteq V$, one may find an open neighborhood $U \subseteq X$ of $x$ such that $\Gamma(u) \subseteq V$ for all $u \in U$. Now let $Z$ be a vector metric space. Then $\Gamma: X \rightarrow 2^{Z}$ is called sequentially strong-weakly closed if $\operatorname{Gr} \Gamma$ is sequentially closed in $X \times Z_{w}$ where $Z_{w}$ is endowed with the weak topology $\sigma\left(Z, Z^{*}\right)$.

Further, $L^{0}(\Omega, E)$ denotes the space of all (equivalence classes of) measurable functions $x: \Omega \rightarrow E$. Given $Z \subset L^{0}\left(\Omega, \mathbb{R}^{m}\right)$, define $Z^{\prime}:=\{\eta \in$ $L^{0}\left(\Omega, \mathbb{R}^{m}\right):\langle\eta, \varphi\rangle_{*} \in \mathbb{R}(\forall \varphi \in Z\}$ where

$$
\langle\eta, \varphi\rangle_{*}:=\int_{\Omega}(\eta(x), \varphi(x)) d x,
$$

and $(\cdot, \cdot)$ is the usual scalar product in the $m$-dimensional Euclidean space $\mathbb{R}^{m}$ equipped the norm $|\cdot|$.

Let $\Phi: \mathbb{R} \rightarrow[0, \infty)$ be some $N$-function (see, e.g., [26, 34]). The Orlicz space is defined by

$$
L_{\Phi}=L_{\Phi}(\Omega, \mathbb{R})=\left\{u \in L^{0}(\Omega, \mathbb{R}): \int_{\Omega} \Phi(\alpha|u(x)|) d x<\infty \text { for some } \alpha>0\right\}
$$

equipped with the Luxemburg norm

$$
\|u\|_{L_{\Phi}}=\inf \left\{\lambda>0: \int_{\Omega} \Phi(|u(x)| / \lambda) d x \leq 1\right\} .
$$

Define

$$
E_{\Phi}=E_{\Phi}(\Omega, \mathbb{R})=\left\{u \in L^{0}(\Omega, \mathbb{R}): \int_{\Omega} \Phi(\alpha|u(x)|) d x<\infty \text { for all } \alpha>0\right\} .
$$

It is known that $E_{\Phi}=L_{\Phi}$ if and only if $\Phi$ satisfies the so-called $\Delta_{2^{-}}$ condition [26]. Further (see, e.g., [26, 34]), $\left(E_{\Phi}\right)^{*} \cong\left(E_{\Phi}\right)^{\prime}=\left(L_{\Phi}\right)^{\prime}$ and $\left(E_{\Phi}\right)^{\prime}=L_{\Phi^{*}}$ with equivalent norms where $\Phi^{*}$ is the $N$-function dual to $\Phi$ and any functional $u^{*} \in\left(E_{\Phi}\right)^{*}$ is identified with some $\eta \in L^{0}(\Omega, \mathbb{R})$ such that $u^{*}(\varphi)=\langle\eta, \varphi\rangle_{*}\left(\forall \varphi \in E_{\Phi}\right)$. We denote by $M[E]$ the Orlicz-Bochner space of $u \in L^{0}(\Omega, E)$ equipped with the norm $\|u\|_{M[E]}:=\|\| u(\cdot)\left\|_{E}\right\|_{M}<\infty$ where

$$
M:=L_{\Phi}(\Omega, \mathbb{R}) \quad \text { or } \quad M:=E_{\Phi}(\Omega, \mathbb{R}) .
$$

Let $U$ be an open subset of a Banach space $E$. If $f: U \rightarrow \mathbb{R}$ is Lipschitz continuous on $U$, then $f$ has Clarke's generalized derivative $f^{\circ}(x ; \cdot)$ :

$$
f^{\circ}(x ; v)=\limsup _{\substack{y \rightarrow x \\ \lambda \downarrow 0}} \frac{f(y+\lambda v)-f(y)}{\lambda} \quad(v \in E) .
$$


The set

$$
\partial_{C} f(x)=\left\{\zeta \in E^{*}:\langle\zeta, v\rangle \leq f^{\circ}(x ; v)(\forall v \in E)\right\}
$$

is called the generalized gradient (Clarke's subgradient) of $f$ at $x$ (then $\left.\partial_{C} f(x) \in \operatorname{CvCp}\left(E_{w^{*}}^{*}\right)[13,24]\right)$.

The symbol $1_{D}$ denotes the characteristic function of a measurable set $D$ and $X_{1} \hookrightarrow X_{2}$ means that the embedding $X_{1} \subset X_{2}$ is continuous.

\section{Existence theorem via the multivalued version of the Leray-} Schauder principle. Let $\Omega$ be a bounded domain in $\mathbb{R}^{2}$, and $f: \Omega \times \mathbb{R}^{m} \rightarrow$ $2^{\mathbb{R}^{m}}$ be a multifunction of two variables $(x, u) \in \Omega \times \mathbb{R}^{m}$. We shall consider the weak solvability of the Dirichlet problem

$$
\left\{\begin{array}{l}
-\Delta_{m} u(x) \in f(x, u(x)) \text { for a.e. } x \in \Omega, \\
u_{\mid \partial \Omega}=0,
\end{array}\right.
$$

where $\Delta_{m}=(\Delta, \ldots, \Delta)$ is the $m$-vector Laplacian.

In what follows, we denote the norm in the Lebesgue space $L_{2}=$ $L_{2}\left(\Omega, \mathbb{R}^{m}\right)$ by $\|\cdot\|_{L_{2}}$. As usual, $H^{1}\left(\Omega, \mathbb{R}^{m}\right)$ is the Sobolev space defined by the norm $\|u\|_{H^{1}}=\|u\|_{L_{2}}+\left\|\nabla_{m} u\right\|_{L_{2}}$, while $H_{0}^{1}=H_{0}^{1}\left(\Omega, \mathbb{R}^{m}\right)$ is the closure of $C_{0}^{\infty}\left(\Omega, \mathbb{R}^{m}\right)$ with respect to this norm. Denote by $H^{-1}=H^{-1}\left(\Omega, \mathbb{R}^{m}\right)$ the dual space to $H_{0}^{1}$. It is known (see, e.g., [20, Section 8.2, Theorem 5.8]) that there exists an invertible continuous linear operator $L: H_{0}^{1} \rightarrow H^{-1}$ with

$$
\langle L u, u\rangle \geq \alpha\|u\|_{H_{0}^{1}}^{2}
$$

for some $\alpha>0$ such that $L u=u^{*}$ if and only if $\left\langle\nabla_{m} u, \nabla_{m} \varphi\right\rangle_{*}=u^{*}(\varphi)$ for all $\varphi \in H_{0}^{1}\left(\Omega, \mathbb{R}^{m}\right)$. Recall that the weak solvability of (3.1) in $H_{0}^{1}$ means the existence of $u \in H_{0}^{1}$ and $\eta \in N_{f}(u)$ such that $\eta \in H^{-1}$ and $L u=\eta$.

From now on, we denote by $X, Y$ and $Z$ the Orlicz spaces

$$
\begin{aligned}
& X=E_{\Phi_{\varepsilon}}\left[\mathbb{R}^{m}\right], \quad Y=L_{\Phi_{\varepsilon}^{*}}\left[\mathbb{R}^{m}\right], \quad Z=L_{\Phi_{\varepsilon}}\left[\mathbb{R}^{m}\right], \\
& \Phi_{\varepsilon}(t)=\exp \left(|t|^{2-\varepsilon}\right)-1, \quad 0<\varepsilon<1 .
\end{aligned}
$$

Theorem 3.1. Let $\Omega \subset \mathbb{R}^{2}$ and let $f: \Omega \times \mathbb{R}^{m} \rightarrow \operatorname{CvCp}\left(\mathbb{R}^{m}\right)$ be a multivalued nonlinearity with the following properties:

(E1) $\quad f(\cdot, u)$ is Sel-measurable for each $u$ and $f(x, \cdot)$ is locally bounded and closed a.e.;

(E2) $\quad N_{f}: X \rightarrow 2^{Y}$ maps any ball $B_{X}(0, r)$ into the set $\mathcal{N}=N_{f}\left(B_{X}(0, r)\right)$ which is bounded and $\sigma(Y, Z)$-weakly equicontinuous, i.e.

$$
\lim _{\operatorname{meas}(D) \rightarrow 0} \sup _{y \in \mathcal{N}} \int_{\Omega}\left|\left(1_{D}(x) y(x), z(x)\right)\right| d x=0 \quad(z \in Z) ;
$$

$\sup \{(u, v): v \in f(x, u)\} \leq a|u|^{2}+b(x)\left(\forall u \in \mathbb{R}^{m}\right)$ a.e. where $0 \leq$ $a<\mu_{\Delta}, b \in L_{1}(\Omega,[0, \infty))$ and $\mu_{\Delta}$ is the first Dirichlet eigenvalue of the Laplacian $-\Delta_{m}$ on $\Omega$. 
Then the Dirichlet problem (3.1) has a weak solution $u_{*} \in H_{0}^{1}\left(\Omega, \mathbb{R}^{m}\right) \subset$ $E_{\Phi_{0}}\left[\mathbb{R}^{m}\right]$ where $\Phi_{0}(t)=\exp \left(t^{2}\right)-1$.

Proof. We shall apply [2, Theorem 2] (the proof of that theorem was based on the multivalued version of the Leray-Schauder principle) together with the scheme of [2, pp. 118-119]. Then it remains to check that $K N_{f}$ : $X \rightarrow \operatorname{CvCp}(X)$ is u.s.c. and compact where $K:=L^{-1}$. We divide this proof into Steps 3.1-3.5. First, we recall some notions [14, 8, 31]. A set $\mathcal{M} \subset Y$ is called sequentially $\sigma(Y, Z)$-weakly pre-compact (or conditionally sequentially $\sigma(Y, Z)$-compact $)$ if each sequence $y_{j} \in \mathcal{M}$ has some $\sigma(Y, Z)$-weak Cauchy subsequence $y_{j(k)}$, that is, $z \in Z \Rightarrow \lim _{k, l \rightarrow \infty}\left\langle y_{j(k)}-y_{j(l)}, z\right\rangle_{*}=0$.

STEP 3.1. Since the embedding $I: H_{0}^{1} \hookrightarrow X$ is compact (by the Pokhozhaev-Trudinger theorem [20, Theorem 7.15, Section 7.8]) and $X^{*} \cong Y$ with equivalent norms $[5,26], J: Y \hookrightarrow H^{-1}$ is compact. Then the operator $K_{\mid Y}=I \circ K \circ J: Y \rightarrow X$ is compact.

STEP 3.2. By (E2), $A:=N_{f}\left(B_{X}\left(u_{0}, r\right)\right)$ is bounded in $Y$ for any $r \in$ $(0, \infty)$. By [28, Proposition 2.1], (E1)-(E2) imply $A \neq \emptyset$. By Step 3.1, $K_{\mid Y} A$ is pre-compact in $X$. Hence, the multivalued operator $K N_{f}: X \rightarrow 2^{X} \backslash\{\emptyset\}$ is compact.

STEP 3.3. We claim that $B:=K N_{f}(u) \in \operatorname{Cp}(X)$ for any $u \in X$. Let $\left\{w_{n}\right\}_{n \in \mathbb{N}} \subset B$. Then $w_{n}=K\left(v_{n}\right)$ for some $v_{n} \in N_{f}(u)$. By (E2), the sequence $v_{n}$ is $\sigma(Y, Z)$-weak equicontinuous, and so sequentially $\sigma(Y, Z)$ weakly pre-compact, due to the Nakano-Dieudonné $\sigma(Y, Z)$-weak pre-compactness theorem $[14,8,31]$ for $Y=Y^{\prime \prime}$ with $Z=Y^{\prime}$. By the NakanoDieudonné $\sigma(Y, Z)$-weak completeness theorem $[14,8,31]$, each $\sigma(Y, Z)$ weakly Cauchy sequence is $\sigma(Y, Z)$-weakly convergent in $Y$ and so there exist a subsequence $n_{j}$ and $v_{0} \in Y$ such that

$$
v_{n_{j}} \rightarrow v_{0} \quad \text { in } \sigma(Y, Z) \text {. }
$$

Since $\Phi_{\varepsilon}^{*}$ satisfies the $\Delta_{2}$-condition [34], we have $Y^{*} \cong Z$ with equivalent norms [5, 26]. By [28, Theorem 2.1] we deduce that the graph $\operatorname{Gr} N_{f}$ is sequentially closed with respect to $\|\cdot\|_{Y} \times \sigma(Y, Z)$. Hence, $v_{0} \in N_{f}(u)$ follows.

By Step 3.1, $K_{1}^{*}: X^{*} \rightarrow Y^{*}, K_{1}:=K_{\mid Y}: Y \rightarrow X$, and so $\left\langle K_{1} v_{n_{j}}, m^{*}\right\rangle=$ $\left\langle v_{n_{j}}, K_{1}^{*} m^{*}\right\rangle$ for $m^{*} \in X^{*}$. Given $m^{\prime} \in X^{\prime} \cong X^{*} \cong Y[5,26]$, we deduce that $K_{1}^{*} m^{\prime} \in Y^{*} \cong Z$ and

$$
\left\langle K_{1} v_{n_{j}}, m^{\prime}\right\rangle_{*}=\left\langle K_{1} v_{n_{j}}, m^{\prime}\right\rangle=\left\langle v_{n_{j}}, K_{1}^{*} m^{\prime}\right\rangle=\left\langle v_{n_{j}}, K_{1}^{*} m^{\prime}\right\rangle_{*} .
$$

By $(3.4),\left\langle v_{n_{j}}, K_{1}^{*} m^{\prime}\right\rangle_{*} \rightarrow\left\langle v_{0}, K_{1}^{*} m^{\prime}\right\rangle_{*}$ for all $m^{\prime} \in X^{\prime}$. Since

$$
\left\langle v_{0}, K_{1}^{*} m^{\prime}\right\rangle=\left\langle K_{1} v_{0}, m^{\prime}\right\rangle=\left\langle v_{0}, K_{1}^{*} m^{\prime}\right\rangle_{*}=\left\langle K_{1} v_{0}, m^{\prime}\right\rangle_{*},
$$


we get $\left\langle K_{1} v_{n_{j}}, m^{\prime}\right\rangle_{*} \rightarrow\left\langle K_{1} v_{0}, m^{\prime}\right\rangle_{*}$ for all $m^{\prime} \in X^{\prime}=Y$, and so

$$
w_{n_{j}}=K_{1}\left(v_{n_{j}}\right) \rightarrow K_{1}\left(v_{0}\right)=: w_{0} \quad \text { in } \sigma\left(X, X^{\prime}\right)=\sigma(X, Y) \text {. }
$$

By Step 3.2, there exist a subsequence $n(j)$ of $n_{j}$ and $\widetilde{w}_{0} \in X$ such that $w_{n(j)} \rightarrow \widetilde{w}_{0}$ in $X$-norm. Hence, $w_{n(j)} \rightarrow \widetilde{w}_{0}$ in measure. By (3.5) and the Hahn-Saks-Vitali theorem [16], we get $\widetilde{w}_{0}=w_{0}=K_{1}\left(v_{0}\right)$. Therefore, $\widetilde{w}_{0} \in B$. Hence, $B$ is compact in $X$.

STEP 3.4. We claim that $K N_{f}$ is closed. Let $\left[u_{n}, w_{n}\right] \in \operatorname{Gr} K N_{f}$ with $u_{n} \rightarrow u_{0}, w_{n} \rightarrow \widetilde{w}_{0}$. Then $w_{n}=K\left(v_{n}\right)$ for some $v_{n} \in N_{f}\left(u_{n}\right)$. By (E2), the sequence $N_{f}\left(u_{n}\right)$ is $\sigma(Y, Z)$-weakly equicontinuous, and then by the NakanoDieudonné $\sigma(Y, Z)$-weak pre-compactness theorem, $\left\{v_{n}\right\}_{n \in \mathbb{N}}$ is sequentially $\sigma(Y, Z)$-weakly pre-compact. By the Nakano-Dieudonné $\sigma(Y, Z)$-weak completeness theorem $\left(Y=Y^{\prime \prime}, Z=Y^{\prime}\right)$, there exist a subsequence $n_{j}$ and $v_{0} \in Y$ such that $v_{n_{j}} \rightarrow v_{0}$ in $\sigma(Y, Z)$. By [28, Theorem 2.1] for the case $Y^{*}=Z$, the graph $\operatorname{Gr} N_{f}$ is sequentially closed with respect to $\|\cdot\|_{Y} \times \sigma(Y, Z)$. So, $v_{0} \in N_{f}\left(u_{0}\right)$. By the same argument such as in Step 3.3, we deduce that $w_{n_{j}}=K\left(v_{n_{j}}\right) \rightarrow K\left(v_{0}\right)=: w_{0}$ in $\sigma\left(X, X^{\prime}\right)=\sigma(X, Y)$. Observe that $w_{n_{j}} \rightarrow \widetilde{w}_{0}$ in measure. By the Hahn-Saks-Vitali theorem, $\widetilde{w}_{0}=w_{0}=K\left(v_{0}\right)$. Hence, $\widetilde{w}_{0} \in K N_{f}\left(u_{0}\right)$, and so $K N_{f}$ is closed.

SteP 3.5. By Steps 3.1-3.4, $K N_{f}: X \rightarrow \operatorname{CvCp}(X)$ is closed and locally compact. Then (see e.g. [3, Lemma 2.9]) $K N_{f}$ is u.s.c.

\section{The calculation of Clarke's subgradient for Lipschitz integral} functionals on open balls of Orlicz spaces. If $g: \Omega \times E \rightarrow \mathbb{R}$ is Lipschitz continuous with respect to the second variable, then $g^{\circ}\left(x, u_{0} ; v\right)$ denotes the Clarke derivative at $u_{0}$ in direction $v$ of the function $u \mapsto g(x, u)$. By [13, $24]$ the function $g^{\circ}(x, u ; v)$ is continuous in $v$. For simplicity, let $\partial_{C} g\left(x, u_{0}\right)$ denote the generalized gradient of $g(x, \cdot)$ at $u_{0}$. The proof of Lemma 4.1 is standard via the known measurable selection theorems [18].

Lemma 4.1. Let $g: \Omega \times E \rightarrow \mathbb{R}$ be a function such that $g(\cdot, u)$ is measurable for any $u \in E$ and $g(x, \cdot)$ is Lipschitz continuous an each ball of $E$ for almost all $x \in \Omega$. Then, given any measurable functions $u, v: \Omega \rightarrow E$, the function $x \in \Omega \mapsto g^{\circ}(x, u(x) ; v(x))$ is measurable.

We shall use the Lebourg theorem [13, Theorem 2.3.7]: Let $f: U \rightarrow \mathbb{R}$ be Lipschitz continuous on an open subset $U$ of $E$. Assume that $U$ contains the convex interval $[v, z]$. Then there exists a point $u \in(v, z)$ such that

$$
f(v)-f(z) \in\left\langle\partial_{C} f(u), v-z\right\rangle .
$$

Let $\mathfrak{A}$ be the $\sigma$-algebra of measurable subsets of $\Omega$. A function $f: \Omega \times F \rightarrow$ $\overline{\mathbb{R}}=: \mathbb{R} \cup\{ \pm \infty\}$ is called a normal integrand if $f(x, \cdot)$ is lower semicontinuous for almost all (a.a.) $x \in \Omega$ and there exists $\Omega_{0}$ with $\operatorname{meas}\left(\Omega \backslash \Omega_{0}\right)=0$ such 
that $f$ is $(\mathfrak{A} \times \mathcal{B}(F), \mathcal{B}(\overline{\mathbb{R}}))$-measurable on $\Omega_{0} \times F$. If $F$ is a Lusin space (e.g. $F=E$ or $\left.F=E_{w^{*}}^{*}\right)$ then by [10, Lemma 1.2.3] every Carathéodory function on $\Omega \times F$ is a normal integrand. If $f$ is a normal integrand on $\Omega \times E$ then the dual convex normal integrand $f^{*}: \Omega \times E_{w^{*}}^{*} \rightarrow \overline{\mathbb{R}}$ is defined by $f^{*}\left(x, u^{*}\right)=$ $\sup \left\{\left\langle u, u^{*}\right\rangle-f(x, u): u \in E\right\}$. Denote by $(M[E])_{\mathrm{s}}^{*}$ the space of singular linear functionals on $M[E]$ (see $[22,23]$ ). Lemma 4.2 below is taken from V. Levin [23, Corollary 1 of Theorem 6.7, p. 216] in the general case, and from A. Kozek [22] in the case of $E^{*}$ separable (in this case, $M^{\prime}\left[E_{w^{*}}^{*}\right]=M^{\prime}\left[E^{*}\right]$ ).

LEMMA 4.2. Let $f$ be a normal convex integrand on $\Omega \times E$ and consider the functional $I_{f}, I_{f}(u):=\int_{\Omega} f(x, u(x)) d x$, on $M[E]$. Suppose that $\operatorname{dom} I_{f}:=\left\{u \in M[E]: I_{f}(u)<\infty\right\} \neq \emptyset$. Then for every $u_{0} \in \operatorname{dom} I_{f}$ the subdifferential $\partial I_{f}\left(u_{0}\right)$ consists of all linear functionals $\lambda \in(M[E])^{*}$ of the form $\lambda(u)=\langle u, y\rangle+\lambda_{\mathrm{S}}(u)(u \in M[E])$ where $y \in M^{\prime}\left[E_{\omega^{*}}^{*}\right] \cap \operatorname{Sel} \partial f\left(\cdot, u_{0}(\cdot)\right)$, $\lambda_{\mathrm{s}} \in(M[E])_{s}^{*}, \lambda_{\mathrm{s}} \in K\left(\operatorname{dom} I_{f}, u_{0}\right):=\left\{l \in(M[E])^{*}: l\left(z-u_{0}\right) \leq 0\right.$ $\left.\left(\forall z \in \operatorname{dom} I_{f}\right)\right\}$, and $\partial f\left(x, u_{0}\right)$ denotes the subdifferential at $u_{0}$ of the convex function $u \mapsto f(x, u)$.

Given $R \in(0, \infty)$, we shall use the following conditions $(\Phi 1)$ and $(\Phi 2)$ :

$(\Phi 1) \quad$ There exists $\Omega_{0} \in \mathfrak{A}$ with meas $\left(\Omega \backslash \Omega_{0}\right)=0$ and there exist $b_{R}, d_{R} \in$ $(0, \infty)$ and $a_{R} \in L_{1}(\Omega,[0, \infty))$ such that

$$
u^{*} \in \partial_{C} g(x, u) \Rightarrow \Phi^{*}\left(\frac{\left\|u^{*}\right\|_{E^{*}}}{d_{R}}\right) \leq a_{R}(x)+b_{R} \Phi\left(\frac{\|u\|_{E}}{R}\right)
$$

for all $x \in \Omega_{0}$ and $u \in E$.

(\$2) There exists $\Omega_{0} \in \mathfrak{A}$ with meas $\left(\Omega \backslash \Omega_{0}\right)=0$ and there exist $b_{R}, d_{R} \in$ $(0, \infty), a_{R} \in L_{1}(\Omega,[0, \infty))$, and $\widetilde{h}_{R}: \Omega \times[0, \infty) \rightarrow[0, \infty)$ such that

$$
|g(x, u)-g(x, v)| \leq \widetilde{h}_{R}(x,|u|+|v|)|u-v|
$$

for all $x \in \Omega_{0}$ and for all $u, v \in E$, and

$$
\Phi^{*}\left(\frac{\widetilde{h}(x, \alpha)}{d_{R}}\right) \leq a_{R}(x)+b_{R} \Phi\left(\frac{\alpha}{R}\right)
$$

for all $x \in \Omega_{0}$ and $\alpha \in[0, \infty)$.

Given a function $g: \Omega \times E \rightarrow \mathbb{R}$, define the integral functional

$$
G(u):=\int_{\Omega} g(x, u(x)) d x .
$$

ThEOREM 4.3. Let $g: \Omega \times E \rightarrow \mathbb{R}$ be a Carathéodory function such that $g(x, \cdot)$ is Lipschitz continuous on each ball of $E$ for almost all $x \in \Omega$. Suppose that either $\partial_{C} g$ satisfies $(\Phi 1)$ or $g$ satisfies $(\Phi 2)$ for some $R \in(0, \infty)$ where $M=L_{\Phi}$ or $M=E_{\Phi}$. 
If $G$ is finite at least for one $\bar{u} \in B_{M[E]}(0, R / 2)$, then $G$ is Lipschitz on $B_{M[E]}(0, R / 2)$ and

$$
u \in \operatorname{Int} B_{M[E]}(0, R / 2) \Rightarrow \partial_{C} G(u) \subset N_{\partial_{C} g}(u),
$$

i.e. $\gamma \in \partial_{C} G(u) \Rightarrow \gamma(\cdot)=\langle\zeta, \cdot\rangle_{*}$ for some $\zeta \in L_{\Phi^{*}}\left[E_{w^{*}}^{*}\right]$ with $\zeta(x) \in$ $\partial_{C} g(x, u(x))$ a.e. If additionally the function $g(x, \cdot)$ is regular (in Clarke's sense) at $u(x)$ for almost all $x \in \Omega$, then the functional $G$ is regular at $u$ and $\partial_{C} G(u)=N_{\partial_{C} g}(u)$.

Proof. We shall mimic Clarke's proof of his Theorem 2.7.5/(B) in [13] but new elements in our proof will be emphasized and given in detail. We divide the proof into Steps 4.1-4.7. Observe that $(\Phi 2)$ implies $(\Phi 1)$. Later on, we suppose $(\Phi 1)$.

STEP 4.1. Let $\left(\Phi^{*}\right)^{-1}(x, \cdot)$ be the right pre-image of $\Phi^{*}(x, \cdot)$. Define $h_{R}: \Omega \times[0, \infty) \rightarrow[0, \infty)$,

$$
h_{R}(x, \alpha):=d_{R}\left(\Phi^{*}\right)^{-1}\left(x, a_{R}(s)+b_{R} \Phi(x, \alpha / R)\right) .
$$

There exists $\Omega_{1} \subset \Omega_{0}$ with meas $\left(\Omega \backslash \Omega_{1}\right)=0$ such that $h_{R}(x, \cdot)$ is nondecreasing for $x \in \Omega_{1}$. Hence,

$$
\sup \left\{\left\|u^{*}\right\|_{E^{*}}: u^{*} \in \partial_{C} g(x, u),\|u\|_{E} \leq a(x)\right\} \leq h_{R}(x, a(x)) \quad \text { a.e. }
$$

for each $a \in B_{M_{+}}(0, R)$ where

$$
M_{+}:=\{u \in M: u(x) \geq 0 \text { a.e. }\} .
$$

Since $M^{\prime}=L_{\Phi^{*}}(\Omega, \mathbb{R})$ with equivalent norms, it is easy to check (see, e.g., [5]) that the superposition operator $N_{h_{R}}: B_{M_{+}}(0, R) \rightarrow M^{\prime}, N_{h_{R}}(a)(x):=$ $h_{R}(x, a(x))$, is bounded.

STEP 4.2. We claim that the operator $N_{h_{R}}$ : Int $B_{M_{+}}(0, R) \rightarrow M^{\prime}$ has the $U$-property, i.e., given any $a \in \operatorname{Int} B_{M_{+}}(0, R)$, for each sequence $\left\{a_{k}\right\} \subset M_{+}$ with

$$
r_{1}:=\|a\|_{M}+\sum_{k=1}^{\infty}\left\|a_{k}-a\right\|_{M}<R
$$

there exists $d \in M^{\prime}$ such that

$$
\left|N_{h_{R}}\left(a_{k}\right)(x)\right| \leq d(x) \quad \text { a.e. on } \Omega \text { for every } k \in \mathbb{N} \text {. }
$$

To prove this, fix a sequence $\left\{a_{k}\right\} \subset M_{+}$such that $r_{1}<R$. Then by the Riesz-Fischer property for the Banach lattice $M$ (see, e.g., [5]; this property for the Orlicz space can be directly deduced from the Lebesgue dominated convergence theorem), there exists $\Omega_{2} \subset \Omega_{1}$ with meas $\left(\Omega \backslash \Omega_{2}\right)=0$ such that the series $a_{\infty}(x):=\sum_{k=1}^{\infty}\left|a_{k}(x)-a(x)\right|$ converges for $x \in \Omega_{2}$; moreover putting $a_{\infty}(x):=0$ for $x \in \Omega \backslash \Omega_{2}$, we get $a_{\infty} \in M_{+}$with $\left\|a_{\infty}\right\|_{M} \leq$ $\sum_{k=1}^{\infty}\left\|a_{k}-a\right\|_{M}<\infty$. Note that $a_{k}(x) \leq a_{\infty}(x)+a(x)$ a.e. and $\left\|a_{k}\right\|_{M} \leq$ 
$r_{1}<R$, and then by Step 4.1 we have

$N_{h_{R}}\left(a_{k}\right)(x)=h_{R}\left(x, a_{k}(x)\right) \leq h_{R}\left(x, a_{\infty}(x)+a(x)\right)=N_{h_{R}}\left(a_{\infty}+a\right)(x)=: d(x)$ a.e. Since $N_{h_{R}}$ maps $B_{M_{+}}(0, R)$ into $M^{\prime}$, we have $N_{h_{R}}\left(a_{\infty}+a\right)=d(\cdot) \in M^{\prime}$.

STEP 4.3. We claim that the functional $G$ is Lipschitz continuous on $B_{M[E]}(0, R / 2)$. In fact, let $y, z \in B_{M[E]}(0, R / 2)$. By Lebourg's theorem [13, Theorem 2.3.7] for $g(x, \cdot)$ on some open ball containing the convex interval $[z(x), y(x)]$, we can find $\xi_{0}(x) \in E_{\omega^{*}}^{*}, u_{0}(x) \in E$ and $\theta_{0}(x) \in[0,1]$ such that

$$
u_{0}(x)=\theta_{0}(x) z(x)+\left(1-\theta_{0}(x)\right) y(x) \quad \text { and } \quad \xi_{0}(x) \in \partial_{C} g\left(x, u_{0}(x)\right)
$$

with

$$
g(x, z(x))-g(x, y(x))=\left\langle\xi_{0}(x), z(x)-y(x)\right\rangle \quad \text { a.e. }
$$

We point out that the functions $\xi_{0}, u_{0}, \theta_{0}$ are not, in general, measurable. Note that

$$
\left\|u_{0}(x)\right\|_{E} \leq\left\|\theta_{0}(x) z(x)\right\|_{E}+\left\|\left(1-\theta_{0}(x)\right) y(x)\right\|_{E} \leq\|z(x)\|_{E}+\|y(x)\|_{E} \text { a.e. }
$$

Therefore,

$$
\begin{aligned}
\|\| z(\cdot)\left\|_{E}+\right\| y(\cdot)\left\|_{E}\right\|_{M} & \leq\|\| z(\cdot)\left\|_{E}\right\|_{M}+\|\| y(\cdot)\left\|_{E}\right\|_{M} \\
& =\|z\|_{M[E]}+\|y\|_{M[E]} \leq R .
\end{aligned}
$$

By Step 4.1, $\gamma_{0}$ defined by $\gamma_{0}(x):=N_{h_{R}}\left(x,\|z(x)\|_{E}+\|y(x)\|_{E}\right)$ satisfies $\left\|\gamma_{0}\right\|_{M^{\prime}} \leq \widetilde{C}(R)$ where $\widetilde{C}(R):=\sup \left\{\left\|N_{h_{R}}(\widetilde{\alpha})\right\|_{M^{\prime}}:\|\widetilde{\alpha}\|_{M} \leq R\right\}<\infty$. Observe that $\left\|\xi_{0}(x)\right\|_{E^{*}} \leq \gamma_{0}(x)$ a.e. Since

$$
|g(x, z(x))-g(x, y(x))| \leq\left\|\xi_{0}(x)\right\|_{E^{*}}\|z(x)-y(x)\|_{E} \quad \text { a.e., }
$$

by the generalized Hölder inequality [26, 34], we have

$$
\begin{aligned}
& |G(z)-G(y)| \leq \int_{\Omega}|g(x, z(x))-g(x, y(x))| d x \\
& \leq \int_{\Omega} \gamma_{0}(x)\|z(x)-y(x)\|_{E} d x \leq\left\|\gamma_{0}\right\|_{M^{\prime}}\|\| z(\cdot)-y(\cdot)\left\|_{E}\right\|_{M} \\
& \leq \widetilde{C}(R)\|z-y\|_{M[E]} \quad \text { for } z, y \in B_{M[E]}(0, R / 2) \text {. }
\end{aligned}
$$

Since $\bar{u} \in B_{M[E]}(0, R / 2)$ with $G(\bar{u}) \in \mathbb{R}$, the claim of Step 4.3 follows.

STEP 4.4. We shall prove that $G^{\circ}(x ; v) \leq \int_{\Omega} g^{\circ}(x, u(x) ; v(x)) d x$ for $u \in$ Int $B_{M[E]}(0, R / 2)$ and $v \in M[E]$. Observe that both sides of this inequality are positively homogeneous in $v$, so it suffices to prove this inequality for $v$ from $B_{M[E]}(0,1)$. From the definition (2.2) and (4.1) we have

$$
G^{\circ}(u ; v)=\limsup _{\substack{y \rightarrow u \\ \lambda \downarrow 0}} \int_{\Omega} \frac{g(x, y(x)+\lambda v(x))-g(x, y(x))}{\lambda} d x .
$$


Choose arbitrary sequences $\lambda_{k}$ in $\mathbb{R}$ and $y_{k}$ in $\operatorname{Int} B_{M[E]}(0, R / 2)$ such that $\lambda_{k} \downarrow 0,\left\|y_{k}-u\right\|_{M[E]} \rightarrow 0$ and the limit

$$
b:=\lim _{k \rightarrow \infty} \int_{\Omega} F_{k}(x) d x
$$

exists, where

$$
F_{k}(x):=\frac{g\left(x, y_{k}(x)+\lambda_{k} v(x)\right)-g\left(x, y_{k}(x)\right)}{\lambda_{k}} .
$$

By [5] together with Riesz's theorem, we can choose a subsequence $k_{j}$ and $D_{0}$ with meas $\left(\Omega \backslash D_{0}\right)=0$ such that $y_{k_{j}}(x) \rightarrow u(x)$ as $j \rightarrow \infty\left(\forall x \in D_{0}\right)$, $\left\|y_{k_{j}}-u\right\|_{M[E]} \leq R / 2^{j+2}$ and $\lambda_{k_{j}} \leq R / 2^{j+2}$.

We claim that there exist $\beta \in L_{1}(\Omega, \mathbb{R})$ and $D_{1} \subset D_{0}$ with meas $\left(\Omega \backslash D_{1}\right)$ $=0$ such that $\left|F_{k_{j}}(x)\right| \leq \beta(x)$ on $D_{1}$ for all $j \in \mathbb{N}$. To prove this, by Lebourg's theorem for $g(x, \cdot)$ on some open ball containing the convex interval $\left[y_{k}(x), y_{k}(x)+\lambda_{k} v(x)\right]$, there exist $\xi_{k}(x) \in E_{w^{*}}^{*}, u_{k}(x) \in E$ and $\alpha_{k}(x) \in[0,1]$ such that

$$
\begin{aligned}
& u_{k}(x)=\alpha_{k}(x)\left[y_{k}(x)+\lambda_{k} v(x)\right]+\left(1-\alpha_{k}(x)\right) y_{k}(x), \\
& g\left(x, y_{k}(x)+\lambda_{k} v(x)\right)-g\left(x, y_{k}(x)\right)=\left\langle\xi_{k}(x), \lambda_{k} v(x)\right\rangle, \\
& \xi_{k}(x) \in \partial_{C} g\left(x, u_{k}(x)\right) \quad \text { a.e. }
\end{aligned}
$$

So $F_{k}(x)=\left\langle\xi_{k}(x), v(x)\right\rangle$. We point out that the functions $\xi_{k}, u_{k}$ and $\alpha_{k}$ are not, in general, measurable. We have

$$
\begin{aligned}
& \left\|u_{k_{j}}(x)\right\|_{E} \\
& \quad \leq\|u(x)\|_{E}+\left\|\alpha_{k_{j}}(x)\left[y_{k_{j}}(x)+\lambda_{k_{j}} v(x)\right]+\left(1-\alpha_{k_{j}}(x)\right) y_{k_{j}}(x)-u(x)\right\|_{E} \\
& \quad \leq\|u(x)\|_{E}+\left\|y_{k_{j}}(x)-u(x)\right\|_{E}+\lambda_{k_{j}}\|v(x)\|_{E}=: a_{j}(x) \quad \text { a.e. }
\end{aligned}
$$

Observe that for the sequence $a_{j}$ and $a, a(x):=\|u(x)\|_{E}$ with $\|a\|_{M}<R / 2$, we get

$$
\begin{aligned}
\left\|a_{j}-a\right\|_{M} & =\|\| y_{k_{j}}(\cdot)-u(\cdot)\left\|_{E}+\left|\lambda_{k_{j}}\right|\right\| v(\cdot)\left\|_{E}\right\|_{M} \\
& \leq\|\| y_{k_{j}}(\cdot)-u(\cdot)\left\|_{E}\right\|_{M}+\left|\lambda_{k_{j}}\right|\|\| v(\cdot)\left\|_{E}\right\|_{M} \\
& =\left\|y_{k_{j}}-u\right\|_{M[E]}+\left|\lambda_{k_{j}}\right|\|v\|_{M[E]} \leq R / 2^{j+2}+R / 2^{j+2}\|v\|_{M[E]},
\end{aligned}
$$

so that

$$
r_{1}=\|a\|_{M}+\sum_{j=1}^{\infty}\left\|a_{j}-a\right\|_{M}<R / 2+R / 2=R .
$$

By Steps 4.1-4.2, $\left\|\xi_{k_{j}}(x)\right\|_{E^{*}} \leq h\left(x, a_{j}(x)\right)=N_{R}\left(a_{j}\right)(x)$ a.e. and there exists $d \in M^{\prime}$ such that $\left|N_{h_{R}}\left(a_{j}\right)(x)\right| \leq d(x)$ a.e. Hence, as $\left|F_{k_{j}}(x)\right| \leq$ $\left\|\xi_{k_{j}}(x)\right\|_{E^{*}}\|v(x)\|_{E}$, we deduce the existence of $D_{1} \subset D_{0}$ with meas $\left(\Omega \backslash D_{1}\right)$ $=0$ such that $\left|F_{k_{j}}(x)\right| \leq d(x)\|v(x)\|_{E}=: \beta(x)\left(\forall x \in D_{1}\right)$. Since $\|v(\cdot)\|_{E} \in M$, we obtain $\beta \in L_{1}(\Omega, \mathbb{R})$. 
Using the above claim together with the measurability of the function $x \mapsto g^{\circ}(x, u(x) ; v(x)$ ) (see Lemma 4.1), we can apply the Fatou lemma to the functions $x \mapsto \beta(x)-F_{k_{j}}(x) \in[0, \infty)$ and deduce that

$$
\begin{aligned}
& b=\limsup _{j \rightarrow \infty} \int_{\Omega} F_{k_{j}}(x) d x \leq \int_{\Omega} \limsup _{j \rightarrow \infty} F_{k_{j}}(x) d x=\int_{D_{1}} \limsup _{j \rightarrow \infty} F_{k_{j}}(x) d x
\end{aligned}
$$

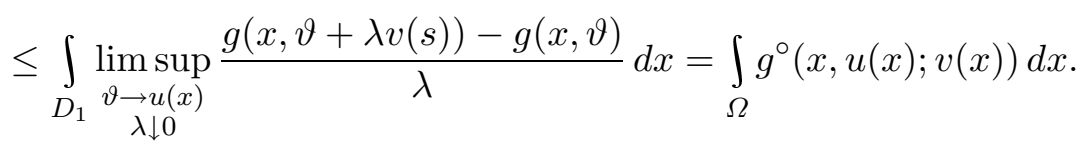

Therefore, by (4.3) and (4.4), we get

$$
\begin{aligned}
G^{\circ}(u ; v) & =\sup \left\{b=\lim _{k \rightarrow \infty} \int_{\Omega} F_{k}(x) d x:\left\|y_{k}-u\right\|_{M[E]} \rightarrow 0, \lambda_{k} \downarrow 0\right\} \\
& \leq \int_{\Omega} g^{\circ}(x, u(x) ; v(x)) d x .
\end{aligned}
$$

STEP 4.5. We claim that the function $x \in \Omega \mapsto g^{\circ}(x, u(x) ; v(x))$ belongs to $L_{1}(\Omega, \mathbb{R})$ for any $u \in \operatorname{Int} B_{M[E]}(0, R / 2)$ and $v \in M[E]$. Again, it suffices to prove this for $v \in B_{M[E]}(0, R / 4)$. Since $M$ is a Banach lattice, by [5] there exists $\alpha \in M$ such that $\|\alpha\|_{M} \leq R / 4$ with $\alpha(x)>0$ for $x \in \operatorname{supp} M=\Omega$. Then, by Lebourg's theorem, Step 4.1 implies that

$$
\begin{aligned}
& \left|g^{\circ}(x, u(x) ; v(x))\right| \\
& \leq \sup \left\{|c|: c=\frac{g(x, \bar{u}+\lambda v(x))-g(x, \bar{u})}{\lambda}, \lambda \in(0,1],\|\bar{u}-u(x)\|_{E} \leq \alpha(x)\right\} \\
& \leq \sup \left\{\left|\left\langle v(x), u^{*}\right\rangle\right|: u^{*} \in \partial_{C} g(x, u), u \in[\bar{u}, \bar{u}+\lambda v(x)], \lambda \in(0,1],\right. \\
& \left.\leq \sup \left\{\left|\left\langle v(x), u^{*}\right\rangle\right|: u^{*} \in \partial_{C} g(x, u),\|u\|_{E} \leq p(x)\right\} \quad\|\bar{u}-u(x)\|_{E} \leq \alpha(x)\right\} \\
& \leq\|v(x)\|_{E} h_{R}(x, p(x)) \quad \text { a.e. }
\end{aligned}
$$

where $p(x):=\|u(x)\|_{E}+\alpha(x)+\|v(x)\|_{E}$ satisfies $p \in M_{+}$with $\|p\|_{M} \leq r_{2}:=\|\| u(\cdot)\left\|_{E}\right\|_{M}+\|\alpha\|_{M}+\|\| v(\cdot)\left\|_{E}\right\|_{M}<R / 2+R / 4+R / 4=R$.

Hence, by Step 4.1, the above claim follows.

SteP 4.6. Fix $u \in \operatorname{Int} B_{M[E]}(0, R / 2)$. We mimic Clarke's argument in the proof of [13, Theorem 2.7.2]. We know (see Lemma 4.1) that the function $(x, \widetilde{v}) \in \Omega \times E \mapsto g^{\circ}(x, u(x) ; \widetilde{v})$ is a Carathéodory convex integrand, and $v \in M[E] \mapsto \widetilde{G}(v):=\int_{\Omega} g^{\circ}(x, u(x) ; v(x)) d x$ is a convex functional on $M[E]$ such that $\widetilde{G}(0)=0$. If $\gamma \in \partial_{C} G(u)$, then by Step 4.4 for every $v \in M[E]$ we have

$$
\gamma(v) \leq G^{\circ}(u ; v) \leq \int_{\Omega} g^{\circ}(x, u(x) ; v(x)) d x=\widetilde{G}(v)-\widetilde{G}(0)
$$


and so $\gamma$ is an element of the subdifferential $\partial \widetilde{G}(0)$. By Lemma 4.2 together with $\operatorname{dom} I_{\widetilde{G}}=M[E]$ (see Step 4.5), we can deduce that $\partial \widetilde{G}(0)$ consists of linear functionals $\gamma \in(M[E])^{*}$ of the form $\langle\gamma, v\rangle=\int_{\Omega}\langle\zeta(x), v(x)\rangle d x$ $(v \in M[E])$ with $\zeta \in M^{\prime}\left[E_{w^{*}}^{*}\right]$ and $\zeta(x) \in \partial_{C} g(x, u(x))$ a.e. on $\Omega$. Hence, the inclusion (4.2) follows.

STEP 4.7. Fix $u \in \operatorname{Int} B_{M[E]}(0, R / 2)$. Suppose that $g(x, \cdot)$ is regular (in Clarke's sense [13, Section 2.3]) at $u(x)$ for a.a. $x \in \Omega$. Fix $v \in M[E]$. By an analogous argument to the one in Step 4.4, we can apply the Fatou lemma to the functions $x \mapsto \beta(x)+F_{k_{j}}(x) \in[0, \infty)$ and deduce that

$$
\begin{aligned}
\liminf _{\lambda \downarrow 0} \frac{G(u+\lambda v)-G(u)}{\lambda} \geq \int_{\Omega} \liminf _{\lambda \downarrow 0} \frac{g(x, u(x)+\lambda v(x))-g(x, u(x))}{\lambda} d x \\
=\int_{\Omega} g^{\prime}(x, u(x) ; v(x)) d x=\int_{\Omega} g^{\circ}(x, u(x) ; v(x)) d x \geq G^{\circ}(u ; v) .
\end{aligned}
$$

Now by Clarke's argument in the proof of [13, Theorem 2.7.3, p. 87] we conclude that $G$ is regular at $u$ and $N_{\partial_{C} g}(u) \subset \partial_{C} G(u)$.

5. Existence theorem via the nonsmooth variational technique. By the Poincaré inequality [20], $\|u\|_{H_{0}^{1}}^{*}=\|\nabla u\|_{L_{2}}$ defines an equivalent norm in $H_{0}^{1}\left(\Omega, \mathbb{R}^{m}\right)$, and so by the Pokhozhaev-Trudinger embedding theorem [20, Theorem 7.15, Section 7.8] there exists $c(\varepsilon) \in(0, \infty)$ such that

$$
\|u\|_{L_{\Phi_{\varepsilon}}\left[\mathbb{R}^{m}\right]} \leq c(\varepsilon)\|\nabla u\|_{L_{2}} \quad\left(\forall u \in H_{0}^{1}\left(\Omega, \mathbb{R}^{m}\right)\right) .
$$

Theorem 5.1. Let $\Omega \subset \mathbb{R}^{2}$ and let $g: \Omega \times \mathbb{R}^{m} \rightarrow \mathbb{R}$ be a Carathéodory function such that $g(x, \cdot)$ is Lipschitz continuous on each ball of $\mathbb{R}^{m}$ for almost all $x \in \Omega$. Assume the Hammerstein condition:

$$
\begin{aligned}
& g(x, u) \leq \frac{1}{2} \gamma|u|^{2}+\delta(x)\left(\forall u \in \mathbb{R}^{m}\right) \text { a.e. where } 0<\gamma<\mu_{\Delta}, \delta \in \\
& L_{1}(\Omega,[0, \infty)), \text { and } \mu_{\Delta} \text { is the first Dirichlet eigenvalue of the Lapla- } \\
& \text { cian }-\Delta_{m} \text { on } \Omega .
\end{aligned}
$$

Moreover, let $\varepsilon \in(0, \infty)$ and either $(\Phi 1)$ or $(\Phi 2)$ be valid for $\Phi(t)=\Phi_{\varepsilon}(t)=$ $\exp \left(|t|^{2-\varepsilon}\right)-1$ with $R:=2 c(\varepsilon) \varrho_{*}$ where $\varrho_{*} \in(0, \infty)$ satisfies

$$
\varrho_{*}^{2}>\frac{2 \mu_{\Delta}}{\mu_{\Delta}-\gamma} \int_{\Omega}(\delta(x)-g(x, 0)) d x \in[0, \infty) .
$$

Then the problem (3.1) with $f(x, u)=\partial_{C} g(x, u) \subset \mathbb{R}^{m}$ has at least one weak solution $u_{*} \in H_{0}^{1}\left(\Omega, \mathbb{R}^{m}\right) \subset E_{\Phi_{0}}\left[\mathbb{R}^{m}\right]$ where $\Phi_{0}(t)=\exp \left(t^{2}\right)-1$.

Proof. We divide this proof into Steps 5.1-5.5. 
STEP 5.1. Define the energy functional

$$
J(u):=\frac{1}{2} \int_{\Omega}|\nabla u|^{2} d x-\int_{\Omega} g(x, u(x)) d x \quad\left(u \in H_{0}^{1}\left(\Omega, \mathbb{R}^{m}\right)\right) .
$$

We claim that $J: B_{H_{0}^{1}}\left(0, \varrho_{*}\right) \rightarrow \mathbb{R}$ is sequentially weakly l.s.c. Fix $u$ with $\|u\|_{H_{0}^{1}}^{*} \leq \varrho_{*}$. By (5.1), $\|u\|_{L_{\Phi_{\varepsilon}}\left[\mathbb{R}^{m}\right]} \leq c(\varepsilon) \varrho_{*}=R / 2$. By Theorem 4.3 for $\Phi=\Phi_{\varepsilon}$ under $(\Phi 1)$ or $(\Phi 2)$ for $R=2 c(\varepsilon) \varrho_{*}, G: B_{X}(0, R / 2) \rightarrow \mathbb{R}$ is Lipschitz continuous. By the Alaoglu-Bourbaki theorem for the reflexive separable space $H_{0}^{1}\left(\Omega, \mathbb{R}^{m}\right), B_{H_{0}^{1}}\left(0, \varrho_{*}\right)$ is sequentially weakly compact. Fix $u_{n} \rightarrow u_{0}$ in $B_{H_{0}^{1}}\left(0, \varrho_{*}\right)$. Since $H_{0}^{1} \hookrightarrow X$ compactly by the Pokhozhaev-Trudinger theorem [20, Theorem 7.15, Section 7.8], the sequence $u_{n}$ is pre-compact in $X$. Hence, there exists a subsequence $n_{j}$ such that $u_{n_{j}} \rightarrow u_{0}$ in $X$-norm. It is known (see, e.g., [17]) that $\liminf _{j \rightarrow \infty} \int_{\Omega}\left|\nabla u_{n_{j}}\right|^{2} d x \geq \int_{\Omega}\left|\nabla u_{0}\right|^{2} d x$. Hence, $\liminf _{n \rightarrow \infty} J\left(u_{n}\right) \geq J\left(u_{0}\right)$, i.e. $J$ is sequentially weakly l.s.c. on $B_{H_{0}^{1}}\left(0, \varrho_{*}\right)$.

SteP 5.2. We claim that $J(0)<J(u)$ for $u \in \partial B_{H_{0}^{1}}\left(0, \varrho_{*}\right)$. Fix $u$ with $\|u\|_{H_{0}^{1}}^{*}=\varrho_{*}$. Then, by (E4) and (E5), we get

$$
\begin{aligned}
J(u) & \geq \frac{1}{2} \int_{\Omega}|\nabla u|^{2} d x-\frac{1}{2} \gamma \int_{\Omega}|u(x)|^{2} d x-\int_{\Omega} \delta(x) d x \\
& \geq \frac{1}{2}\left(1-\frac{\gamma}{\mu_{\Delta}}\right) \int_{\Omega}|\nabla u|^{2} d x-\int_{\Omega} \delta(x) d x \\
& =\frac{1}{2} \frac{\left(\mu_{\Delta}-\gamma\right) \varrho_{*}^{2}}{\mu_{\Delta}}-\int_{\Omega} \delta(x) d x>J(0) .
\end{aligned}
$$

STEP 5.3. From Step 5.1, by [17, Theorem 1.1], $J$ attains its minimum on $B_{H_{0}^{1}}\left(0, \varrho_{*}\right)$ at some $u_{*} \in B_{H_{0}^{1}}\left(0, \varrho_{*}\right)$. By Step 5.2, $u_{*} \in \operatorname{Int} B_{H_{0}^{1}}\left(0, \varrho_{*}\right)$, and by (5.1), $u_{*} \in \operatorname{Int} B_{X}(0, R / 2)$. By [13, Proposition 2.4.11], we infer that $0 \in \partial_{C} J\left(u_{*}\right) \subset H_{0}^{-1}\left(\Omega, \mathbb{R}^{m}\right)=\left(H_{0}^{1}\left(\Omega, \mathbb{R}^{m}\right)\right)^{*}$.

STEP 5.4. By (4.1) and (5.2), we have $J(u)=J_{1}(u)-J_{2}(u)$ where $J_{1}(u):=\frac{1}{2} \int_{\Omega}|\nabla u|^{2} d x, J_{2}(u):=G_{\mid H_{0}^{1}}(u)=(G \circ P)(u)$, and $P: \operatorname{Int} B_{H_{0}^{1}}\left(0, \varrho_{*}\right)$ $\hookrightarrow X$. The functional $J_{1}$ is continuously Fréchet differentiable, i.e. $J_{1} \in C^{1}$ and $J_{1}^{\prime}(u)(\cdot)=\langle L u, \cdot\rangle$ (see [17]). By [15, Lemma 2.1], the set $C_{0}^{\infty}\left(\Omega, \mathbb{R}^{m}\right) \subset$ $H_{0}^{1}\left(\Omega, \mathbb{R}^{m}\right)$ is dense in $X$. By [12], [13, Proof of Part 3 of Theorem 2.3.10, p. 46], [13, Corollary in p. 47] or [11, Theorem 2.2] for $u_{*} \in \operatorname{Int} B_{H_{0}^{1}}\left(0, \varrho_{*}\right)$ (see Step 5.3), we have $\partial_{C}(G \circ P)\left(u_{*}\right)=\partial_{C} G\left(u_{*}\right)$. Hence, by [13, Proposition 2.3.3, Proposition 2.3.1, Corollary 1, Proposition 2.2.4] together with Theorem 4.3 for $u_{*} \in \operatorname{Int} B_{X}(0, R / 2)$ (see Step 5.3), we get

$$
\begin{aligned}
\partial_{C} J\left(u_{*}\right) & =\partial_{C} J_{1}\left(u_{*}\right)+\partial_{C}\left(-J_{2}\left(u_{*}\right)\right)=J_{1}^{\prime}\left(u_{*}\right)-\partial_{C} J_{2}\left(u_{*}\right) \\
& =J_{1}^{\prime}\left(u_{*}\right)-\partial_{C} G\left(u_{*}\right) \subset J_{1}^{\prime}\left(u_{*}\right)-N_{\partial_{C} g}\left(u_{*}\right)=L u_{*}-N_{\partial_{C} g}\left(u_{*}\right) .
\end{aligned}
$$


SteP 5.5. By Steps 5.3-5.4, $0 \in L u_{*}-N_{\partial_{C} g}\left(u_{*}\right)$, which is equivalent to $L u_{*} \in N_{\partial_{C} g}\left(u_{*}\right)$. By the assumption $f(x, \cdot)=\partial_{C} g(x, \cdot), u_{*}$ is a weak solution of (3.1).

REMARK 5.2. By the proof of Theorem 5.1, the statement of Theorem 5.1 remains valid if we assume instead of (E4) any verifiable condition that implies the coerciveness of $J$ (i.e. $J(u) \rightarrow \infty$ as $\|u\|_{H_{0}^{1}} \rightarrow \infty$ ). Many such conditions can be found in [1]. Further, Theorems 3.1 and 5.1 have analogs for the Dirichlet inclusion involving a general uniformly elliptic operator.

Acknowledgements. The authors are grateful for the constructive remark of the reviewer which influenced the shape of the revised version.

\section{References}

[1] A. Anane and J.-P. Gossez, Strongly nonlinear elliptic problems near resonance. A variational approach, Comm. Partial Differential Equations 15 (1990), 1141-1159.

[2] J. Appell, E. De Pascale, H. T. Nguyêñ and P. P. Zabrejko, Nonlinear integral inclusions of Hammerstein type, Topol. Methods Nonlinear Anal. 5 (1995), 109-122.

[3] - , - - - -, Multivalued superpositions, Dissertationes Math. 345 (1995).

[4] J. Appell, H. T. Nguyêñ, and P. P. Zabrejko, Multivalued superposition operators in ideal spaces of vector functions I, Indag. Math. (N.S.) 2 (1991), 385-395.

[5] J. Appell and P. P. Zabrejko, Nonlinear Superposition Operators, Cambridge Univ. Press, Cambridge, 1990.

[6] R. Bader, A topological fixed-point index theory for evolution inclusions, Z. Anal. Anwendungen 20 (2001), 3-15.

[7] G. Bartuzel and A. Fryszkowski, A class of retracts in $L^{p}$ with some applications to differential inclusion, Discuss. Math. Differ. Incl. Control Optim. 22 (2002), 213-224.

[8] O. Burkinshaw and P. Dodds, Weak sequential compactness and completeness in Riesz spaces, Canad. J. Math. 28 (1976), 1332-1339.

[9] T. Cardinal and N. S. Papageorgiou, Hammerstein integral inclusions in reflexive Banach spaces, Proc. Amer. Math. Soc. 127 (1999), 95-103.

[10] C. Castaing, P. Raynaud de Fitte and M. Valadier, Young Measures on Topological Spaces with Applications in Control Theory and Probability Theory, Kluwer, Dordrecht, 2004.

[11] K.-C. Chang, Variational methods for non-differentiable functionals and their applications to partial differential equations, J. Math. Anal. Appl. 80 (1981), 102-129.

[12] F. H. Clarke, Generalized gradients of Lipschitz functionals, Adv. Math. 40 (1981), $52-67$.

[13] -, Optimization and Nonsmooth Analysis, SIAM, Philadelphia, 1983.

[14] J. Dieudonné, Sur les espaces de Köthe, J. Anal. Math. 1 (1951), 81-115.

[15] T. K. Donaldson and N. S. Trudinger, Orlicz-Sobolev spaces and imbedding theorems, J. Funct. Anal. 8 (1971), 52-75.

[16] N. Dunford and J. T. Schwartz, Linear Operators, Part I: General Theory, Interscience, New York, 1957.

[17] D. G. de Figueiredo, Lectures on the Ekeland Variational Principle with Applications and Detours, Springer, Berlin, 1989. 
[18] A. Fryszkowski, Fixed Point Theory for Decomposable Sets, Kluwer, Dordrecht, 2004.

[19] L. Gasiński and N. S. Papageorgiou, Nonsmooth Critical Point Theory and Nonlinear Boundary Value Problems, Chapman \& Hall/CRC, London, 2004.

[20] D. Gilbarg and N. Trudinger, Elliptic Partial Differential Equations of Second Order, Springer, New York, 1983.

[21] S. Hu and N. S. Papageorgiou, Handbook of Multivalued Analysis: Applications, Vol. II, Kluwer, Dordrecht, 2000.

[22] A. Kozek, Convex integral functionals, Comment. Math. Prace Mat. 21 (1980), 109138.

[23] V. L. Levin, Convex Analysis in Spaces of Measurable Functions and its Applications in Mathematics and Economics, Nauka, Moscow, 1985 (in Russian).

[24] B. S. Mordukhovich, Variational Analysis and Generalized Differentiation. Vol. I: Basic Theory, Vol. II: Applications, Springer, Berlin, 2005.

[25] D. Motreanu and V. Rădulescu, Variational and Non-Variational Methods in Nonlinear Analysis and Boundary Value Problems, Kluwer, Dordrecht, 2003.

[26] J. Musielak, Orlicz Spaces and Modular Spaces, Lecture Notes in Math. 1034, Springer, Berlin, 1983.

[27] H. T. Nguyêñ, On Banach function M-spaces, Indag. Math. (N.S.) 14 (2002), 551564 .

[28] - On the sequential strong-weak closedness of the Nemytskij multivalued operator, Demonstratio Math. 35 (2002), 365-374.

[29] —, Semicontinuity and continuous selections for the multivalued superposition operator without assuming growth-type conditions, Studia Math. 163 (2004), 1-19.

[30] H. T. Nguyêñ, M. Juniewicz and J. Ziemińska, CM-selectors for pairs of oppositely semicontinuous multifunctions and some applications to strongly nonlinear inclusions, Z. Anal. Anwendungen 19 (2000), 1-12.

[31] M. Nowak, Conditional and relative weak compactness in vector-valued function spaces, J. Convex Anal. 12 (2005), 447-463.

[32] R. Płuciennik, S. Tian and Y. Wang, Non-convex integral functionals on MusielakOrlicz spaces, Comment. Math. Prace Mat. 30 (1990), 113-123.

[33] R. Precup, Fixed point theorems for decomposable multi-valued maps and applications, Z. Anal. Anwendungen 22 (2003), 843-861.

[34] M. M. Rao and Z. D. Ren, Theory of Orlicz Spaces, Dekker, New York, 1991.

Hông Thái Nguyêñ

Institute of Mathematics

Szczecin University

Wielkopolska 15

70-451 Szczecin, Poland

E-mail: nguyenht@sus.univ.szczecin.pl congdungthai@yahoo.com
Dariusz Pączka Institute of Mathematics Szczecin Technical University Al. Piastów 48/49 70-310 Szczecin, Poland E-mail: paczka@ps.pl,paczdar@yahoo.com

Received October 24, 2005; 